\title{
Graves' disease combined with acute myocarditis and thyrotoxic periodic paralysis in a male:a case report and review of the literature
}

\author{
Jun Teng ${ }^{1}$, Weisheng Liu ${ }^{1}$, and Ruicai Shan ${ }^{1}$ \\ ${ }^{1}$ Qingdao Center Hospital
}

January 13, 2022

\begin{abstract}
Graves' disease is the most common reason for hyperthyroidism which manifest as multi-system changes.Among these clinical manifestations acute myocarditis and thyrotoxic periodic paralysis are very rare and patients who are combined with both two haven't been reported yet.The etiology may attribute to autoimmunity.

Graves' disease combined with acute myocarditis and thyrotoxic periodic paralysis in a male:a case reportand review of the literature

Mengmei Li,MD ${ }^{\mathrm{a}}$,Weisheng Liu $\mathrm{MD}^{\mathrm{a}}, \mathrm{PingLi}^{\mathrm{a}}$, RuicaiShan, $, \mathrm{MD}^{\mathrm{b}}, \mathrm{Jun} \mathrm{TengMD}^{\mathrm{a}}$

a:Emergency Department of Qingdao Central Hospital,Qingdao,Shandong,China.

b:Utrosonography Department of Qingdao Central Hospital, Shandong,China.

Shorttitle:Graves' disease combined with acute myocarditis and thyrotoxic periodic paralysis

Corresponding Author:

Full name:Jun Teng

Email:owen-145@163.com

Emergency Department,Qingdao Central Hospital,Qingdao,Shandong,China.
\end{abstract}

Abstract:

BACKGROUND

Acute myocarditis is an acute myocardium injury that manifests as arrhythmia, dyspnea, and elevated cardiac enzymes. Acute myocarditis is usually caused by a viral infection but can sometimes be caused by autoimmunity. Graves' disease is an autoimmune disease that is a rare etiology of acute myocarditis. Accelerated junctional rhythm is also a rare manifestation of acute myocarditis in adults.

\section{CASE SUMMARY}

A rare case of new-onset Graves' disease combined with acute myocarditis and thyrotoxic periodic paralysis is reported. The patient was a 25-year-old young man who suddenly became paralyzed and felt palpitations and dyspnea. He was then sent to our emergency department (ED). Upon arrival, electrocardiography revealed an accelerated junctional rhythm and ST-segment depression in all leads, and laboratory findings showed 
extreme hypokalemia and elevated troponin I, with the troponin I level being $0.32 \mathrm{ng} / \mathrm{mL}$ (reference range, 0-0.06 ng/mL). Coronary computer tomography angiography was performed, and there were no abnormal findings in the coronary arteries. Subsequently, the patient was admitted to the ED ward, where further testing revealed Graves' disease, along with continued elevated cardiac enzyme levels and B-type natriuretic peptide (BNP) levels. The troponin I level was $0.24 \mathrm{ng} / \mathrm{mL}$ after admission. All of the echocardiography results were normal: Left atrium $35 \mathrm{~mm}$, left ventricle $48 \mathrm{~mm}$, end-diastolic volume $102 \mathrm{~mL}$, right atrium 39 $\mathrm{mm} \times 47 \mathrm{~mm}$, right ventricle $25 \mathrm{~mm}$, and ejection fraction $60 \%$. Cardiac magnetic resonance was performed on the fifth day of admission, revealing myocardial edema in the lateral wall and intramyocardial and subepicardial late gadolinium enhancement in the lateral apex, anterior lateral, and inferior lateral segments of the ventricle. The patient refused to undergo an endomyocardial biopsy. After $6 \mathrm{~d}$, the patient's cardiac enzymes, BNP, potassium, and electrocardiography returned to normal. After the patient's symptoms were relieved, he was discharged from the hospital. During a 6-mo follow-up, the patient was asymptomatic and subjected to thyroid function, liver function, kidney function, troponin I, and electrocardiograph routine tests for medicine adjustments. The hyperthyroid state was controlled.

\section{CONCLUSION}

Acute myocarditis is a rare manifestation of Graves' disease. Accelerated junctional rhythm is also a rare manifestation of acute myocarditis in adults. When the reason for hypokalemia and elevated cardiac enzymes in patients is unknown, cardiologists should consider Graves' disease and also pay attention to accelerated junctional rhythm.

Key words: Graves'diease,myocarditis,thyrotoxic periodic paralysis,junctional tachycardia.

Key clnical message:

Physicians should not ignore Graves' disease when facing patients unknown paralysis.Physicians should know acute myocarditis is a rare combination of Graves' disease which etiology may contribute to autoimmunity.

\section{Introduction:}

We report a case of acute myocarditis and thyrotoxic periodic paralysis in the setting of new-onset Graves' disease. A previously healthy 25-year-old male presented with sudden paralysis,palpitations,dyspnea,elevated troponin I and extremehypokalemia.After thorough examination he was diagnosed with Graves' disease combined with acute myocarditis and thyroxic periodic paralysis.

I

2 Case report

.Graves' disease is an autoimmune disorder that affects the thyroid gland ${ }^{[1]}$. Hyperthyroidism affects $0.5 \%$ $2 \%$ of females ${ }^{[2]}$ in geographical areas not featuring iodine deficiency. Males show a 10 -fold lower prevalence. Graves' disease is the most frequent cause and is more likely to occur in female populations ${ }^{[2]}$. Graves' disease would seem to be more frequent in Asian populations and less frequent in sub-Saharan Africans ${ }^{[2]}$. Thyroid hormone $(\mathrm{TH})$ receptors are present in the myocardium and vascular endothelial tissues, thereby allowing changes in circulating TH concentration to modulate end-organ activity ${ }^{[3]}$. Thus, Graves' disease can present with cardiovascular manifestations. Usually misdiagnosed as myocardial infarction, Graves' disease combined with acute myocarditis is a rare manifestation, and the etiology is due to an autoimmune process.

When the electrical activity of the sinoatrial node is blocked or is less than the automaticity of the atrioventricular node/His bundle, a junctional rhythm originates ${ }^{[4]}$. Numerous conditions can cause a junctional rhythm, among which myocarditis is a rare etiology ${ }^{[4]}$. Acute myocarditis should be diagnosed when several differential diagnoses are excluded, such as tachycardiomyopathy (TCMP), stress cardiomyopathy, and pericardial diseases. Acute myocarditis presents with junctional arrhythmia is reported in children and seldomly 
reported in adults. There have been a few reports about Graves' disease combined with acute myocarditis ${ }^{[5-7]}$. However, the patient's manifestations differ in these cases. None of these cases presents with junctional arrhythmia. In this case, the patient presented with an accelerated junctional rhythm and myocarditis, which is unique compared with other reported cases, so that clinicians can have a new understanding of the cardiovascular complications of Graves' disease.

A 25-year-old young male realized that he was paralyzed when he woke in the morning. At the same time, the patient felt palpitations, dyspnea, and nausea, with one instance of vomiting gastric contents. The patient was then brought to the emergency department (ED) by ambulance. Upon arrival, electrocardiography revealed an accelerated junctional rhythm (heart rate 91 beats per minute, Figure 1) and ST-segment depression in all leads. The laboratory results showed potassium $1.7 \mathrm{mmol} / \mathrm{L}$ and troponin I $0.32 \mathrm{ng} / \mathrm{mL}$ (reference range, $0-0.06 \mathrm{ng} / \mathrm{mL}$ ). Acute myocardial infarction or acute myocarditis and hypokalemic periodic paralysis were considered, and thus, the ED administered potassium supplements orally and intravenously, oxygen inspiration, aspirin, and clopidogrel. Metoprolol was administered to control the heart rate. Due to the young age of the patient and no risk factors contributing to acute myocardial infarction, the ED department suggested an emergent coronary computer tomography (CT) angiography and a brain computer tomography to rule out more dangerous diseases. The results showed no abnormal findings of the coronary artery and the brain. Accordingly, the patient was diagnosed with acute myocarditis. The patient was then admitted to the ED ward, in which he was diagnosed with suspected acute myocarditis and hypokalemic periodic paralysis (reason unknown). The next step was to determine the primary disease.

\section{History of past illness}

The patient had no previous health issues.

\section{Personal and family history}

The patient's family history did not reveal anything significant to the present condition. The patient was healthy and had not taken any drugs previously. He also reported no recent changes in weight.

\section{Physical examination}

The patient was conscious and afebrile, and his blood pressure was 110/65 mmHg. He was agitated and sweating profusely. Muscle strength was grade 2. According to the patient's high metabolic condition, hyperthyroidism was considered the most common cause of hypokalemic periodic paralysis in young males. We especially checked the thyroid gland. There was no exophthalmos of the patient's eyes, and no restriction of eye movements. There were no hand tremors. Palpation of the thyroid showed II degree of swelling of the thyroid gland with no abnormal findings on the isthmus. There was no tenderness. On auscultation of the thyroid, a bruit could be heard. The lungs, heart, and abdomen were subsequently examined, all of which were normal.

\section{Laboratory examinations}

Thyroid function tests revealed a hyperthyroid state, and thus, Graves' disease was considered: T3 17.51 pmol/L (3.1-6.8 pmol/L), T4 $39.68 \mathrm{pmol} / \mathrm{L}(12-22 \mathrm{pmol} / \mathrm{L})$, thyroid-stimulating hormone (TSH) 0.005 $\mu \mathrm{IU} / \mathrm{mL}(0.27-4.2 \mu \mathrm{IU} / \mathrm{mL}$ ), thyroglobulin $94.77 \mathrm{ng} / \mathrm{mL}$, anti-thyroglobulin antibodies $18.35 \mathrm{IU} / \mathrm{mL}$ (normal), TSH receptor antibody (TSHR-AB) 13.76 IU/L (0-1.5 IU/L), and thyroid peroxidase antibody 77.67 $\mathrm{IU} / \mathrm{mL}(0-34 \mathrm{IU} / \mathrm{mL})$. Other significant laboratory findings revealed elevated troponin I and elevated B-type natriuretic peptide (BNP) [troponin I $0.24 \mathrm{ng} / \mathrm{mL}$ (reference range, 0-0.06 ng/mL) and BNP $196.24 \mathrm{pg} / \mathrm{mL}$ ]. 
The troponin I level measurement was performed five times, and the trend is shown in Figure 2. The inflammatory markers C-reactive protein and erythrocyte sedimentation rate were also measured, which were elevated to $12.6 \mathrm{mg} / \mathrm{L}$ and $50.3 \mathrm{~mm} / \mathrm{h}$, respectively (the references were within $0.5 \mathrm{mg} / \mathrm{L}$ and $20 \mathrm{~mm} / \mathrm{h}$, respectively). Initially, viral myocarditis was considered. The nucleic acids of 13 common virus types were checked in throat swabs and no positive results were found. The 13 virus types were as follows: Adenovirus, influenza-a, influenza-b, parainfluenza virus, respiratory syncytial virus, Bocavirus, rhinovirus, influenza H1N1, chlamydia, metapneumovirus, influenza H3N2, coronavirus, and Mycoplasma pneumoniae. Since the belief was that autoimmunity might be the etiology, cardiac magnetic resonance (CMR) and endocardial myocardial biopsy (EMB) were suggested.

\section{Imaging examinations}

CMR was performed on the fifth day of admission. The results showed myocardial edema in the lateral wall and intramyocardial and subepicardial late gadolinium enhancement in the lateral apex, anterolateral, and inferior lateral segments of the ventricle (Figure 3 and 4). Said results suggested acute myocarditis. The patient refused to undergo an EMB examination, but echocardiography was performed, with the results being normal: Left atrium $35 \mathrm{~mm}$, left ventricle (LV) $48 \mathrm{~mm}$, end-diastolic volume $102 \mathrm{~mL}$, right atrium $39 \mathrm{~mm} \times 47 \mathrm{~mm}$, right ventricle $25 \mathrm{~mm}$, and ejection fraction (EF) $60 \%$. Thyroid ultrasonography was performed to confirm the diagnosis of Graves' disease, which showed an enlarged thyroid gland and rich blood flow signal, and no tumor was found. Thyroid static imaging was then performed to exclude subacute thyroiditis, which showed bilateral lobe swelling and increased function. Such examinations confirmed the diagnosis of Graves' disease.

\section{FINAL DIAGNOSIS}

Acute myocarditis presenting as an accelerated junctional rhythm in Graves' disease.

\section{TREATMENT}

An endocrinologist was consulted, who suggested that the patient should undergo radioactive iodine therapy. However, the patient expressed a preference for taking medicine. Thus, according to recommendations, methimazole $20 \mathrm{mg} / \mathrm{d}$ was administered to treat hyperthyroidism, while trimetazidine $60 \mathrm{mg} / \mathrm{d}$, metoprolol $50 \mathrm{mg} / \mathrm{d}$, and calcium dibutyryl adenosine cyclophosphate $40 \mathrm{mg} / \mathrm{d}$ were administered for myocarditis.

\section{OUTCOME AND FOLLOW-UP}

The patient's symptoms were relieved within $6 \mathrm{~d}$, and troponin I, BNP, and electrocardiography tests were performed. All tests showed normal results (Figure 5). The patient was discharged from the hospital and was instructed to continue taking methimazole, trimetazidine, and metoprolol.

A 6-mo follow-up process was performed in the emergency clinic and by phone calls, and the patient continued taking metoprolol, trimetazidine, and thiamazole. The patient was asymptomatic aside from several symptoms of thyrotoxicosis, and subjected to thyroid function, liver function, kidney function, troponin I, and electrocardiograph routine tests for medicine adjustments. After $45 \mathrm{~d}$, all of the patient's symptoms disappeared and thyroid function improved: T3 $12.26 \mathrm{pmol} / \mathrm{L}(3.1-6.8 \mathrm{pmol} / \mathrm{L})$, T4 $28.37 \mathrm{pmol} / \mathrm{L}$ (12-22 $\mathrm{pmol} / \mathrm{L})$, and TSH $0.07 \mu \mathrm{IU} / \mathrm{mL}(0.27-4.2 \mu \mathrm{IU} / \mathrm{mL})$. After $80 \mathrm{~d}$, the euthyroid state was restored, and the patient's liver and kidney functions were in good condition. Electrocardiography and troponin I levels were also normal. Methimazole was adjusted to $5 \mathrm{mg} / \mathrm{d}$ and metoprolol was adjusted to $23.75 \mathrm{mg} / \mathrm{d}$. 


\section{DISCUSSION}

Acute myocarditis is an acute injury of the myocardium that manifests as arrhythmia, dyspnea, and elevated cardiac enzymes. Acute myocarditis is usually caused by a viral infection but can sometimes be caused by autoimmunity. An autoimmune state is always triggered in patients with acute autoimmune myocarditis, such as systemic lupus erythematosus, rheumatoid arthritis, and others ${ }^{[8]}$. Graves' disease is also an autoimmune disease and can manifest as acute autoimmune myocarditis. However, acute autoimmune myocarditis is rarely observed in patients with Graves' disease. Despite a previous case report in which acute autoimmune myocarditis could have been a manifestation of Graves' disease ${ }^{[5]}$, the patient did not manifest with junctional arrhythmia and was not suffering from new-onset Graves' disease. Thus, there are several significant differences in comparison with the present case report. The rarity and diagnosis of this case are further clarified in Tables 1 and $2^{[8]}$.

The present patient's electrocardiograph, elevated troponin I, normal coronary arteries, symptoms, and CMR results were consistent with acute myocarditis ${ }^{[8]}$. However, several differential diagnoses, such as TCMP, stress cardiomyopathy, and pericardial diseases, had to be excluded. If there is evidence of persistent or frequently occurring tachycardia or frequent premature ventricular complexes, the possibility of TCMP should be considered when eliciting a history of any new diagnosis of LV dysfunction. The traditional clinical presentation includes symptoms and signs of congestive heart failure and dilated cardiomyopathy. Other factors that point to a diagnosis of TCMP include: (1) Evidence of a previously normal EF and a degree of LV dysfunction out of proportion to other comorbidities; (2) no other cause of non-ischemic cardiomyopathy (e.g., hypertension, alcohol or drug use, and stress (3) absence of left ventricular hypertrophy; (4) relatively normal LV dimensions (LV end-diastolic dimension below $5.5 \mathrm{~cm}$ ); (5) recovery of LV function after control of tachycardia (by rate control, cardioversion or radiofrequency ablation within 1-6 mo); and (6) rapid decline in LV ejection fraction following the recurrence of tachycardia in a patient with recovered LV function after previous control of tachycardia ${ }^{[9]}$. The patient had no previous health issues and had no history of tachycardia. Moreover, the patient's heart rate was $91 \mathrm{bpm}$ initially, which could not be defined as tachycardia. Hence, there was no evidence of persistent tachycardia. Echocardiography and CMR did not reveal any LV dysfunction. The ejection fraction was normal, and there were no significant abnormalities in the cardiac structure. No dilation of the atrium and ventricles was observed, and no hypertrophy was observed. The results above could exclude the possibility of $\mathrm{TCMP}^{[9]}$. The patient did not meet the criteria for stress cardiomyopathy listed in the guidelines of the Heart Failure Association-European Society of Cardiology Criteria and the Revised Mayo Clinic Criteria ${ }^{[10]}$. The patient did not have left ventricular dysfunction, wall motion abnormalities, or emotional disorders, and echocardiography was normal. The patient's CMR confirmed the diagnosis of myocarditis, which excluded the probability of stress cardiomyopathy ${ }^{[10]}$. According to the latest diagnostic criteria ${ }^{[11]}$, acute pericarditis could be excluded in the patient. The patient did not have chest pain, and a pericardial friction rub was not heard. There was no new ST-segment elevation or PR segment depression in the patient, and CMR results did not suggest pericardial involvement. Since myopericarditis has myocardial involvement, the clinical presentation thereof is considerably similar to that of myocarditis. Myopericarditis was diagnosed when the patient had both acute pericarditis and elevated myocardial injury biomarkers. As aforementioned, acute pericarditis was excluded in the patient, and CMR did not show pericardial involvement. As the primary disease in this patient was myocarditis, myopericarditis could also be excluded ${ }^{[1]}$. According to the latest diagnostic criteria, EMB should be performed, but the patient refused this procedure. The patient's myocarditis was deduced to be attributed to autoimmunity. Treatment of primary diseases is of vital importance. The differential diagnostic process of this case is further clarified in Table 3.

Junctional arrhythmia, including accelerated junctional rhythm and junctional tachycardia, is rarely seen in patients with myocarditis. If the patient's heart rate does not exceed $100 \mathrm{bpm}$, such conditions can be referred to as an accelerated junctional rhythm. No related reports on acute myocarditis and accelerated junctional rhythm were found, but there were reports on junctional tachycardia, usually seen in infants and children. Junctional tachycardia is also known as junctional ectopic tachycardia (JET), and the mechanism thereof is the same as accelerated junctional rhythm. Junctional tachycardia is thought to arise from the 
atrioventricular node and the His bundle area ${ }^{[12]}$. The incessant form of junctional ectopic tachycardia with 1:1 ventriculoatrial conduction, is a regular, short RP, narrow complex tachycardia and similar to typical Atrial Ventricular Nodal Reentry Tachycardia ${ }^{[12]}$. The patient's electrocardiography findings were consistent with an accelerated junctional rhythm, which is rarely seen in children with acute viral myocarditis and even rarer in adults. There has been one report of junctional tachycardia in a child ${ }^{[13]}$. The etiology of accelerated junctional rhythm in the present patient could be attributed to autoimmunity (Table 3).

Graves' disease manifests as a hyperthyroid state but is also an autoimmune process. Based on the patient's thyroid function tests, hyperthyroidism was diagnosed. Measurements of the serum levels of TRAb and thyroid ultrasonography are the most important diagnostic tests for Graves' disease. Following the latest guidelines $^{[14]}$, the patient had high TSHR-AB, and thyroid static imaging further confirmed the diagnosis of Graves' disease. Graves' disease treatment includes radioactive iodine (RAI), antithyroid drugs, and thyroidectomy ${ }^{[15,16]}$. For the present patient, thyroidectomy was not suitable. The patient had acute myocarditis and thyrotoxic periodic paralysis, and RAI was more suitable for rapidly controlling the patient's hyperthyroid state. Attempts were made to persuade the patient to accept RAI, but he and his family opted for treatment by medicine. Thus, in accordance with the guidelines and the endocrinologist's suggestions, methimazole was administered, and the thyroid function was routinely checked. The patient was advised to accept radioactive iodine therapy if methimazole could not control his hyperthyroid state. The diagnosis and treatment of the diseases are further clarified in Table 3.

A limitation of the present case is that EMB was not performed. Current guidelines recommend EMB only in a limited number of clinical scenarios that do not include some common presentations of myocarditis, particularly pseudo-infarction ${ }^{[8]}$. The guidelines give the highest levels of recommendations for EMB in life-threatening clinical manifestations ${ }^{[8]}$. The patient's symptoms of myocarditis were atypical. Therefore, he met the indications for EMB according to the guidelines. Although the patient was advised to accept EMB, the patient still refused. EMB could have provided a definite diagnosis for the patient and been especially beneficial in defining the type of myocarditis. According to the latest guidelines ${ }^{[8]}$, since CMR has a good correlation with EMB, the patient could be diagnosed with myocarditis according to CMR, and other diseases could be excluded. The main issue is that EMB can be beneficial in defining the type of myocarditis, in terms of being autoimmune or viral. However, the treatment of the patient was not primarily affected. According to the latest guidelines of myocarditis, conventional therapy is the same in all types of myocarditis. New treatment methods include anti-viral therapy or immunosuppressive therapy, but the patient did not show any sign of viral infection. Hence, anti-viral treatment was not necessary. The patient was diagnosed with Graves' disease, and autoimmune myocarditis could not be excluded. Thus, the treatment of primary disease was of vital importance. The patient recovered quickly after his symptoms of hyperthyroidism were controlled. EMB was beneficial for the patient, but the patient did not accept this procedure. EMB would be better for diagnosis but would not have primarily affected this case. As such, the decision of the patient was ultimately accepted after failing to persuade him.

In the present case, the patient had an accelerated junctional rhythm, which is significantly rare in adults and is a manifestation of acute myocarditis. The etiology may be attributed to autoimmunity, and cardiologists should not ignore such arrhythmia. From the present patient, autoimmune diseases such as Graves' disease can also cause acute myocarditis in addition to viruses. Thus, cardiologists should not ignore such endocrine diseases.

\section{CONCLUSION}

Usually seen in young males, Graves' disease can manifest as thyrotoxic periodic paralysis, in which sudden paralysis and extreme hypokalemia will be experienced. The correction of hypokalemia and hyperthyroidism will relieve the symptoms. The electrocardiograph of an accelerated junctional rhythm usually shows an absence of $\mathrm{P}$ waves and a heart rate within 60-100 rates per minute. Accelerated junctional rhythm is a manifestation of acute myocarditis. Clinicians should not ignore endocrine diseases when facing patients 
with cardiac manifestations.

Author contributions

Jun Teng contributed to the final version of this report,other authors contributed to the diagnosis and treatment for the patient in the report.

Conflict of interest:

None of the authors have conflict of interest to declare in this article.

Acknowledgement:

None.

Ethical approval:

We have told the patient about the publication of his disease.He agreed and signed the patient consent form.

Funding information:

None.

Reference:

1. Luigi Bartalena. Diagnosis and Management of Graves Disease: A Global Overview.Nat Rev Endocrinol. 2013 Dec;9(12):724-34.

2. Anupam Kotwal, Marius Stan.Current and Future Treatments for Graves' Disease and Graves' Ophthalmopathy.HormMetab Res.2018 Dec;50(12):871-886.

3.Antonelli, A., Fallahi, P., Elia, G., Ragusa, F., Paparo, S. R., Ruffilli, I., . . . Ferrari, S. M. (2020). Graves' disease: Clinical manifestations, immuAlessandro Antonelline pathogenesis (cytokines and chemokines) and therapy. Best Pract Res Clin Endocrinol Metab, 101388.

4. Glass J, Osipoff J. Thyrotoxic periodic paralysis presenting in an African-American teenage male: case report. Int J Pediatr Endocrinol. 2020;2020:7.

5. Miqdad Haider, Aijaz Zeeshan Khan Chachar, Atif Munir. Thyrotoxic Periodic Paralysis.J Ayub Med Coll Abbottabad. Oct-Dec 2019;31(4):619-621.

6. Matthew Correia, Mark Darocki, Eva Tovar Hirashima. Changing Management Guidelines in Thyrotoxic Hypokalemic Periodic Paralysis.J Emerg Med. 2018 Aug;55(2):252-256.

7.Alida L P Caforio, Sabine Pankuweit, Eloisa Arbustini.,... Current State of Knowledge on Aetiology, Diagnosis, Management, and Therapy of Myocarditis: A Position Statement of the European Society of Cardiology Working Group on Myocardial and Pericardial Diseases.Eur Heart J. 2013 Sep;34(33):2636-48, 2648a-2648d.

8.Lancaster ST, Koons KL, Lee YJ, Mazimba S, Kwon Y.Acute autoimmune myocarditis as a manifestation of Graves' disease: A case report and review of the literature.Clin Case Rep. 2019 Jun 24;7(8):1489-1493.

Figure1: Electrocardiograph showed junctional tachycardia(no sinus P waves was found, the heart rate was 91 times per minute which was within 60-130 times per minute),ST depression was seen in all leads.Junctional tachycardia could be seen in patients with acute myocarditis.

Figure 2 Thyroid static imaging results:15 minutes after injection of 99mTc-O4-7mCi,the bilateral thyroid gland were swelling and the function was increased which was consistent with Graves' disease. 